\title{
DESARROLLO REGIONAL TARDÍO Y OCUPACIÓN INCA EN LA PRE-CORDILLERA DE TACNA
}

Jesús Gordillo Begazo'

\begin{abstract}
Sobre la base de un trabajo de reconocimienlo y exploraciones arqueológicas en los valles pre-cordilleranos de Sitajara, Yabroco y Susapaya (provincia de Tarata), realizado durante las dos primeras semanas de noviembre de 1984, se describen dos de los cuatro asentamientos aldeanos registrados; ambos con información cultural para las épocas de los Desarrollos Regionales Tardios y ocupación Inca. Introducimos algunos datos sobre antecedentes arqueológicos actuales y se esboza una aproximación al análisis histórico de los asentamientos ubicados en los valles pre-cordilleranos.
\end{abstract}

\section{ANTECEDENTES Y ALGUNAS CONSIDERACIONES GEOGRAFICAS PREVIAS}

Los trabajos de investigación arqueológica realizados en Tacna, para los periodos culturales planteados en el presente escrito, se desarrollaron fundamentalmente en el litoral y los valles costeros hasta tocar algunos espacios de las primeras estribaciones cordilleranas tacneñas. Los registros nos "hablan" de la presencia de varios sitios que identifican a poblaciones con conocimiento amplio de la cerámica, textilería, agricultura, domesticación de animales, pesca, arte rupestre y arquitectura.

Gracias a los trabajos pioneros de Max Uhle (1919), Junius Bird (1943), Isabel Flores (1969) y de Hermann Trimborn (1975), conocemos de asentamientos agro-alfareros (desde Tiwanaku hasta la época Inca), que activan contactos intensos con poblaciones del altiplano. Se reconocen estilos locales como Chiribaya, San Miguel, Pocoma y Gentilar, que ocupan diversos espacios aprovechables de los valles de Caplina, Sama y Locumba. En el norte de Chile estos estilos son ubicados fundamentalmente en los valles de Lluta, Azapa y Camarones. En todos ellos han dejado huellas contundentes de sus obras dentro de un marco de conducta social múltiple.
Para la Sierra de Tacna los reportes sobre información arqueológica son escasos. Tenemos conocimiento de los trabajos desarrollados por Jorge C. Muelle (1969) y Rogger Ravines (1967) en las cuevas de Toquepala y el Abrigo de Caru; asímismo, son relevantes los esfuerzos preliminares de registro arqueológico realizados por Isabel Flores en la zona de Tarata.

Los mecanismos socio-económicos, políticos, culturales y religiosos, que se dieron entre poblaciones de costa y sierra para el Desarrollo Regional Tardio en Tacna (1000 - 1445 años d.C.), aún no los conocemos realmente. Según los investigadores ariqueños, en la sierra del norte chileno surgieron diversos asentamientos que activaron aldeas de recintos rectangulares, construidos con piedra pircada y que mantenian una intercomunicación con poblaciones de costa y altiplano hasta ahora no muy bien explicada (Muñoz, 1982).

Al sumar estas observaciones, el Instituto Nacional de Cultura de Tacna, dentro de un plan trazado inicia un trabajo de reconocimiento arqueológico de la zona alto-andina del departamento de Tacna. A partir del mes de noviembre de 1984. comienzan los trabajos de exploración y con ello las acciones previas del futuro Catastro e Inventario de los sitios arqueológicos del departamento.

La pre-cordillera o región quechua de Tacna, se define a partir de los 2,800 a 3,500 metros sobre el nivel del mar, con una topografia accidentada de 
profundas y estrechas quebradas, variedad de microclimas, valles con virtudes para el desarrollo agricola y ganadero surcados por ríos de regular caudal (originados por los deshielos del Huacune, Barroso, Yucamani y Tutupaca), que en sus cortos recorridos en la condición de tributarios van a formar parte de las cuencas hidrográficas de los rios Caplina, Sama y Locumba. El clima es templado por lo general, siendo frígido en los meses de junio a agosto y lluvioso de diciembre a marzo.

En los tiempos actuales se mantiene un espacio agricola activo de aproximadamente 9,500 hás con cultivos de maiz, papa, oca, habas, orégano, alfalfa, hortalizas, árboles frutales, tuna, zapallo y otros productos altrernativos. Aprovechan para la actividad agrícola los espacios planos, terrenos de suave pendiente y escarpadas laderas que son habilitadas mediante terrazas o andenes. En alturas sobre los 4,100 m.s.n.m. la presencia de pastizales, bofedales, lagunas y pequeños rios posibilitan la crianza y el pastoreo de alpacas y llamas; además, generan ecosistemas con recursos empleados para la alimentación y la actividad doméstica cotidiana. Aún es posible observar en la zona de puna gran variedad de aves acuáticas y algunos grupos de vicuñas, guanacos, tarucas, suris, zorros, vizcachas, y pumas. La flora está representada por el ichu, tola, queñoa, yareta y otras plantas de uso medicinal como la chijura y el cactus blanco.

\section{RECONOCIMIENTO ARQUEOLÓGICO EN EL VALLE DE SITAJARA}

Se decide reconocer inicialmente el valle de Sitajara, ubicado sobre los $3,139 \mathrm{~m}$ de altura, en el ámbito de influencia del cauce del rio Salado y la unión con el rio Quenasani. Políticamente pertenece al distrito del mismo nombre, provincia de Tarata y departamento de Tacna. También se reconocieron los valles de Yabroco y Susapaya, ubicándose en este último dos aldeas importantes: Kanamarca y Huankarani.

Sitajara forma parte de la cabecera de la cuenca hidrográfica del río Sama. El poblado actual está ubicado en una terraza fluvial a unos $150 \mathrm{~m}$ de altura sobre el fondo del valle; mantiene un patrón urbano tipo «damero» con ciertos rasgos del esquema tradicional de los asentamientos andinos precordilleranos. Se intercomunica por una trocha carrozable con los poblados inmediatos de Yabroco y Susapaya. La misma ruta, a la altura de Challahuaya, se enlaza con la vía carrozable Tacna-TarataCandarave. El acceso a los pequeños caseríos alejados se realiza por caminos de tierra o enlozados (senderos sinuosos), muchos de ellos de posible data pre-hispánica.

De acuerdo al censo de 1981, Sitajara tenia una población de 347 habitantes que explotaban agrícolamente un promedio de 110 hás de terreno. En 1993, el censo arrojó una población de 300 habitantes. La actividad económica, el clima y la fisiografía son comunes y compatibles en términos generales a las caracteristicas de la región quechua de la zona sur del pais y del extremo norte de Chile.

El reconocimiento arqueológico realizado en Sitajara, permitió registrar varios sitios. En este trabajo, nos ocuparemos de dos ubicados estratégicamente en ambos extremos del valle: Capanique y Pukara.

\section{SITIO ARQUEOLÓGICO "CAPANIQUE"}

\section{UBICACIÓN}

Se encuentra ubicado entre las coordenadas $1722^{\prime} 40^{\prime \prime}$ de latitud sur y 70 07'25" de longitud oeste, a una altura de 3,250 m.s.n.m. Ocupa la cima emplanada y parte de ladera del cerro Capanique, a unos $1,000 \mathrm{~m}$ al SW del poblado de Sitajara. Muy cerca, en dirección Este, se desplaza la carretera afirmada Tarata-Susapaya y un camino de tierra de probable data pre-hispánica. Topográficamente se sitúa en la tercera terraza fluvial del perfil del valle, otorgándole al sitio una amplia visión del espacio circundante.

\section{ESTADO DE CONSERVACIÓN}

El sitio arqueológico se encuentra en mal estado de conservación. Su proximidad al pueblo y a las áreas de cultivos, permitieron que los colindantes desbasten gradualmente las piedras de las estructuras o muros del asentamiento arqueológico, con el propósito de reutilizarlas en la construcción de corrales y pircas que delimitan los linderos de las actuales parcelas agricolas. Sólo quedan las bases de algunos muros en espacios planos y de ladera, que denuncian un modelo de estructuras de planta rectangular.

\section{ACCESIBILIDAD Y CONTEXTO ECOLÓGICO}

Se accede al sitio arqueológico fácilmente por el sector Este, a través de un pequeño sendero que se desprende de la carretera afirmada TarataSusapaya. Desde el pueblo de Sitajara podemos llegar al sitio a paso pausado en un promedio de 20 minutos.

Capanique, de acuerdo a la configuración topográfica definida por la ONERN (1976:76), se ubica 
en la tercera área caracterizada por la presencia de montañas y colinas per-áridas, de relieve semiaccidentado, con cerros de mediana elevación en la mayoria de los casos y la presencia de pequeñas mesetas. La ecología del sitio está representada por cactáceas rastreras y algunas gramineas frágiles. Se asocia inmediatamente a la segunda y primera área, aptas para el cultivo agrícola y en donde la vegetación de cuenca es frondosa y la existencia de fauna privilegia una rica variedad de aves. El recurso hídrico está sustentado por el río Salado y la canalización directa desde manantiales y estribaciones cordilleranas. Las laderas -convertidas en andenesy áreas planas han sido incorporadas en gran medida al ejercicio agrario logrando activar un promedio de 110 hás de terreno fértil.

\section{EL MODELO DE ASENTAMIENTO}

La distribución de la arquitectura habitacional en Capanique, está en función de copar espacios accesibles y de amplitud visual. En la emplanada superior se han construido un promedio de 15 estructuras que, pese al mal estado de conservación, es posible advertir la presencia de recintos de piedra escogida y planta rectangular, con muros exteriores a dos hileras e interiores a una sola hilera de piedra unida con barro. Son recintos con dimensiones entre $5 \mathrm{~m} \times 3 \mathrm{~m}$ y $3 \mathrm{~m} \times 2 \mathrm{~m}$. En el sector de laderas las estructuras cubren una pequeña área por lo que suponemos un número mayor de recintos en el sitio. Son terrazas domésticas con muros de contención poco visibles debido al desbaste que ha sido objeto; en algunos casos, los muros sólo conservan la primera hilada de pirca y en otros el desbaste ha sido total.

A escasos metros del conjunto se asocia un grupo de estructuras de adobe con muros de hasta $60 \mathrm{~cm}$ de ancho por $2.00 \mathrm{~m}$ de alto. Los muros están enlucidos y pintados en color amarillo-ocre. Son cuartos de planta rectangular, que conforman una unidad de tres recintos y en su interior mantienen evidencias de restos de excremento de animal (asnos, mulas o caballos ?). Este detalle nos permite pensar en la posibilidad de que se trate de un tambo de data colonial (?), o quizás incaico (?). Pegado al sitio se desplaza un sendero que, según los lugareños, viene desde el altiplano puneño.

Los pobladores de Sitajara, suelen Ilamar "Casa de los españoles" a este conjunto de estructuras de adobe; alusión que, por el momento, resulta tolerable, en la medida que las estructuras no manifiestan rasgos de manufactura contemporánea. Sin embargo, es importante un trabajo de excavación en sus espacios interiores y exteriores para definir con claridad su funcionalidad y filiación cronológica.

\section{EL EQUIPO DOMÉSTICO}

En la superficie del sitio, es común observar concentraciones de cerámica fragmentada esparcida en la cima y en las terrazas que caen al valle, y varios batanes que denuncian la naturaleza doméstica del sitio.

Se realizó un muestreo controlado de cerámica, atendiendo a la naturaleza del asentamiento (una unidad ocupacional). La muestra consistió en la recolección de 44 fragmentos de superficie, de los cuales 14 corresponden a bordes ( $7 \mathrm{c} / \mathrm{d}$ y $7 \mathrm{~s} / \mathrm{d}$ ), 34 a fragmentos de cuerpo (30 c/d y $4 \mathrm{~s} / \mathrm{d}$ ) y dos bases sin decoración.

Para realizar el exámen y la clasificación de dicha muestra, se consideraron 28 fragmentos representativos por grupo y frecuencia. Para el análisis de la cerámica, se utilizó la metodología propuesta por Lumbreras, (Gaceta Arqueológica No 13). EI análisis arrojó los siguientes resultados:

A. La primera clase: correspondiente a las vasijas cerradas; están representadas por fragmentos de cántaros que, en su totalidad, pertenecen a bordes y cuerpos decorados de aribalos. El grosor de los fragmentos es de 3 a $5 \mathrm{~mm}$. Los bordes son expandidos y de labios vicelados. Por otro lado, esta clase está representada por fragmentos de bases planas, bordes y cuerpos decorados $y / n o$ decorados que pertenecen a jarras esféricas pequeñas y regulares. Son de cuello corto y ancho, con bordes ligeramente expandidos y labios redondeados.

B. La segunda clase: corresponde a vasijas abiertas, representadas por un conjunto de fragmentos de cuencos profundos y anchos. Son de dimensiones distintas, cuyo diámetro de la boca oscila entre los 10 y $14 \mathrm{~cm}$; y la profundidad entre los 3.5 y $4.5 \mathrm{~cm}$

En cuanto a la producción alfarera, el análisis macroscópico de la muestra permitió detectar seis grupos o alfares. Mediante este procedimiento se tratará de definir "unidades de producción tecnológicamente uniformes, de cuya unidad se infieren hábitos productivos y condiciones de trabajo iguales y, por lo tanto, asociados a una entidad comunal o especial, tipo taller, que no necesariamente debe coincidir con las clases de artefactos, cuyos usuarios no son necesariamente quienes los produjeron" (Lumbreras, 1987: 4 y 31). 


\section{a) Grupo 1}

Se emparenta con el estilo denominado "Cusco Polícromo B" (para la época Inca). Tiene la pasta muy compacta y homogénea, color rojo naranja que indica una perfecta cocción oxidante; las inclusiones de arena y mica son muy finas. El alisamiento interno es horizontal (generalmente en el caso de las vasijas cerradas) y el externo no se distingue debido a la aplicación de un buen engobe; sin embargo, en algunos fragmentos de cántaros, se observa un alisamiento horizontal. En el caso de la superficie externa de las vasijas abiertas, es notable un tratamiento mixto del alisado.

El acabado está definido por la secuencia: engobe, bruñido, pulido y aplicación decorativa con diseños pintados. En la decoración de las vasijas cerradas predominan los diseños dentados en negro sobre rojo y los helechos. También son importantes los conjuntos de líneas rectas paralelas pintadas en negro sobre rojo y/o naranja, alternadas con guesas bandas color marrón-guinda. Por su parte, la aplicación del color blanco aparece ocasionalmente a manera de líneas o puntos. La decoración en general es de aplicación externa.

En las vasijas abiertas (cuencos, platos y escudillas), predomina los diseños naturalistas pintados en negro sobre rojo.

\section{b) Grupo 2}

Representado por fragmentos de tres jarras y un cuenco vinculado a la cerámica altiplánica Inca conocida como Chucuito Polícromo. Tiene la pasta compacta y homogénea con inclusiones de mica y como temperantes. En el trabajo de alisado y en la aplicación del engobe, mantiene cierta similitud con la cerámica del Grupo 1; más no en el bruñido y pulido por ser muy débil.

Cocción oxidante irregular. Algunos fragmentos de jarras tienen pasta color grisáseo motivada por la deficiencia de cocción. Los fragmentos restantes mantienen una regular cocción oxidante.

\section{c) Grupo 3}

Cerámica de pasta compacta y homogénea, color rojo-naranja e inclusiones finas de mica, arena y otros. Acabado interior y exterior bien logrado; mantiene un alisado horizontal interior muy fino, sobre el cual se aplicó engobe rojo para luego ultimar con un excelente bruñido y pulido. Este grupo está representado por un fragmento (borde) de cuenco, caracterizado por tener una decoración interna a base de un conglomerado de llamitas estilizadas. A este estilo cerámico se le conoce como Saxamar, para el Norte Grande de Chile; y en el altiplano puneño se denomina Chucuito negro sobre rojo. Rogger Ravines reconoce como cerámica Inca Pacaje.

\section{d) Grupo 4}

Representado por un fragmento de cuenco con decoración interna de un diseño circular ovalado con punto concéntrico. Su estructura tecnológica es similar a la cerámica del grupo 3. Por el detalle del tipo de decoración de la cerámica, podríamos vincularla al estilo altiplánico tardío conocido como Kollau(?).

e) Grupo 5

Cerámica de pasta semi-compacta, color anaranjado-rosáceo, con inclusiones granulosas de arena. Buena cocción oxidante. El acabado está definido por un alisado irregular sobre el que se aplica un diluído engobe. La decoración es sobre la base de diseños lineales en colores rojo, anaranjado y marrón oscuro.

Este Grupo está representado por dos fragmentos de jarras pequeñas, emparentados al estilo regional costero Pocoma.

\section{f) Grupo 6}

Representado por tres fragmentos de cuencos, cuatro de jarras y una base. Pasta color café, semi-compacta, no homogénea y con inclusiones de cales, micas, cuarzo y arena. Cocción oxidante incompleta. Superficie estriada, alisamiento irregular y aplicación de un simulado baño de pintura roja-ocre diluída. La cerámica no tiene engobe y en algunos casos son visibles las huellas dactilares del alfarero.

Tecnológicamente, este grupo difiere, sin duda, de los demás alfares; por lo que podría tratarse de un estilo alfarero local.

Asociados a la cerámica y estructuras arquitectónicas, ubicamos en el sitio fragmentos de varios morteros y dos batanes completos registrados en el interior de los recintos. 


\section{SITIO ARQUEOLÓGICO "PUKARA"}

\section{UBICACIÓN}

Se ubica al Noreste del poblado actual de Sitajara, entre las coordenadas $1721^{\prime} 38^{\prime \prime}$ a $1721^{\prime} 45^{\prime \prime}$ de Latitud Sur y 70 06'45" a $7007^{\prime} 11^{\prime \prime}$ 'de Longitud Oeste; y a una altitud de 3,458 m.s.n.m.

Pukara, está enclavado en la cima del cerro del mismo nombre, se levanta a partir de la tercera terraza o tercer nivel del perfil de la cuenca, que se inicia en el lecho del río Salado. Dista 2,300 metros de distancia del pueblo actual de Sitajara.

Ocupa estratégicamente la cima del cerro que es ligeramente plana y alargada, parte de la ladera Suroeste y un nivel inferior hacia el sector Norte. Desde ese punto se domina ampliamente el valle y la confluencia de los ríos Yabroco y Quenasani.

\section{ESTADO DE CONSERVACIÓN}

El sitio arqueológico de Pukara presenta un regular estado de conservación. Las estructuras habitacionales aún conservan una altura importante en los muros; sin embargo, el sector funerario es el que más deteriodo a sufrido, debido al saqueo de chullpas y tumbas soterradas.

\section{ACCESIBILIDAD Y CONTEXTO ECOLÓGICO}

Se accede a Pukara mediante dos vias: a) Carretera afirmada que se desplaza por las faldas del cerro Pukara rumbo a Yabroco y Susapaya, y b) Acceso directo mediante un camino pre-hispánico que se desplaza por el lado SE del moderno cementerio del pueblo y a escasos metros del complejo arqueológico.

El contexto ecológico es similar al del sitio Capanique, con la salvedad que en Pukara se observa un terreno más árido, proliferando las cactáceas rastreras y una gramínea pequeña y frágil. Su acceso al fondo del valle es más prolongado que en Capanique.

El recurso hídrico está sustentado gracias a la presencia del río Salado o Yabroco y a las aguas temporales que trae la quebrada de Quilavira.

\section{EL MODELO DE ASENTAMIENTO}

En Pukara, se observa un formal asentamiento aldeano que ordena su espacio en un marco extremadamente asociado con un sector doméstico predominante de valor estratégico en el valle.

El modelo define claras unidades funcionales, hecho que nos permite describir y explicar de manera ordenada los atributos del sitio arqueológico:

\section{A. Distribución de la arquitectura de hábitat}

Sobre la cima del cerro Pukara y parte de sus laderas, se construyeron un conjunto de estructuras habitacionales, mediando tin trabajo previo de aterrazamiento de los espacios ocupados. La estructura de los muros de contención, varía de acuerdo al nivel de ubicación de las terrazas, que en su conjunto, dan forma a un asentamiento que tiene como promedio 80 recintos.

Son estructuras de planta rectangular y casi cuadrada, distribuidas ordenadamente sobre las terrazas. Sus dimensiones oscilan entre los 5.30 por $3.70 \mathrm{~m}$ y 4.00 por $3.60 \mathrm{~m}$. En el interior de los recintos existen estructuras pequeñas de $1.20 \mathrm{~m}$ por $80 \mathrm{~cm}$ que podrian tratarse de colcas 0 depósitos. Las puertas de acceso a los recintos habitacionales tienen un ancho promedio entre 60 a $70 \mathrm{~cm}$.

Los muros exteriores de las habitaciones generalmente forman parte del muro de contención de la terraza. Son muros construídos de doble hilera con piedras irregulares unidas con mortero de tierra húmeda. En cuanto a los muros interiores, éstos son casi siempre de una sola hilera de piedras. En ambos casos, son muros de 40 y $50 \mathrm{~cm}$ de espesor, salvo los recintos pequeños cuyos muros bordean los $20 \mathrm{~cm}$ de ancho Las uniones o vértices (esquina de los cuartos), son amarrados en ángulo recto hacia el exterior y curvo al interior.

El aparejo de los muros es generalmente irregular, empleándose piedras de distinto tamaño y forma (anguladas y redondeadas); este detalle, sumado al barro utilizado como mortero para la unión de las piedras, hace que el muro mantenga un amarre confiable que le otorga estabilidad (soporte) prudente a los edificios. Los espacios originados en el aparejo de los muros, son rellenados con tierra húmeda y en algunos casos mezclada con piedras menudas.

El conjunto habitacional descrito tiene un área de $2,300 \mathrm{~m}^{2}$. que copa la parte del extremo Oeste del cerro. A 10 metros del grupo habitacional, en dirección Este (parte central de la cima del cerro), se levanta una estructura de piedra de planta rectangular con $8 \mathrm{~m}$ de largo por $6 \mathrm{~m}$ de ancho $y$ muros de hasta $50 \mathrm{~cm}$ de alto. En la parte central del recinto, se ha construido un "altar" de piedra pircada en forma de cono truncado y con hornacinas 
en la parte media. Los pobladores actuales de Sitajara lo utilizan para sus prácticas religiosas alusivas al culto de la cruz. Sobre el "altar", los lugareños mantienen una cruz cristiana ornada con flores y ofrendas.

\section{B. Mecanismo de defensa del hábitat}

Hacia el extremo Este de la cima del cerro, que significa la entrada al sitio habitacional, existe una zanja o fosa de $3 \mathrm{~m}$ de profundidad por $3.30 \mathrm{~m}$ de ancho, que prácticamente se convierte en un verdadero sistema de defensa de la aldea. Se asocian a la fosa dos estructuras que pudieron haber funcionado como puestos de control y vigilancia.

El "corte", dejó al descubierto la estructura sólida del cerro, compuesta por un material de roca volcánica color rosado.

La fosa separa tácitamente al conjunto habitacional de un importante sector de enterramientos, ubicado en la plataforma del cerro.

\section{Espacio de enterramientos}

Está ubicado en un plano inferior del cerro, a 80 metros al Este del conjunto habitacional. Ocupa un área de $820 \mathrm{~m}^{2}$ aproximadamente, en donde se concentran dos sectores de tumbas o tipos de enterratorios: Chullpas y tumbas soterradas.

C. 1 Las Chullpas.- Son ocho chullpas las registradas. Su disposición conserva un alineamiento intensionado, sobre una suave lomada anexa a la plataforma del cerro Pukara. De acuerdo a las características estructurales de las chullpas, éstas han sido ordenadas en dos tipos:

Tipo I.- Chullpas construidas de arcilla color rojiza, de estructura voluminosa y compacta. Tienen planta rectangular y en algunos casos alcanzan hasta los tres metros de altura. Hemos registrado tres chullpas, dos de las cuales se encuentran en regular estado de conservación y una tercera ha sido casi destruida en su totalidad.

La técnica de construcción es en base a sucesivas camadas de paja brava (ichu), alternadas por "tortas" o vaciados de arcilla de hasta $12 \mathrm{~cm}$ de grosor; y asi, continuadamente hasta lograr el volumen, altura y la forma deseada. Son de estructura compacta, con una cámara abovedada de un metro de altura y una pequeña entrada en la base de 40 por $40 \mathrm{~cm}$, mirando al Este.
Como consecuencia de la profanación y destrución parcial de las chullpas, existen fragmentos de huesos y de cerámica diseminados al interior y exterior de las mismas.

Tipo II.- Son de planta circular, con un diámetro de $1.50 \mathrm{~m}$ a $3.00 \mathrm{~m}$ y se ubican alternadamente entre las Chullpas del tipo I. Han sido construidas en base a dos hileras de piedra escogida, unidas con barro y que en su estado original habrian alcanzado la altura promedio de dos metros; hoy, los muros de las chullpas solo tienen de 40 a 50 $\mathrm{cm}$ de altura. Indudablemente, el proceso de devastación a la que han sido sometidas, sólo permite observar las dos o tres primeras hiladas de piedra.

Al igual que en el tipo I, se asocian al interior $y$ exterior de las estructuras funerarias varios fragmentos de cerámica y de óseos humanos, producto de la profanación de las mismas.

C. 2 Tumbas soterradas.- Se caracterizan por estar cavadas bajo la superficie y utilizar paramentos. A este tipo de tumbas se les denomina "cistas". Están ubicadas a 40 metros al NE de la concentración de chullpas y mantienen un patrón distributivo aglutinado, con grupos de 4 y 6 tumbas.

Son tumbas de planta circular, con un diámetro promedio del $60 \mathrm{~cm}$ y una profundidad que oscila entre los 70 y $90 \mathrm{~cm}$. Los paramentos son de piedra escogida, unidas con barro y se alzan en hileras sucesivas hasta alcanzar el borde al nivel de la superficie. La cubierta o tapa de las tumbas, son de dos tipos: a) una o dos lajas planas de origen volcánico y b) amontonamiento ordenado de piedras regulares que cierran la entrada de la tumba, con la ayuda de tierra húmeda, resultando un terminal abovedado de la cubierta.

El cementerio aún se encuentra en buen estado de conservación, siendo poca la cuota de tumbas "huaqueadas", lo que permite observar sus detalles estructurales y algunos fragmentos de cerámica y restos óseos en el interior y fuera de las tumbas. Se realizó un trabajo de limpieza en una de las tumbas, cuya información facilitó ampliar la descripción de las cistas y la identificación contextual de la alfarería.

\section{EL ESPACIO AGRICOLA}

Pukara está rodeado por un conjunto de andenes abandonados en casi su totalidad. Los actuales lugareños dinamizan algún sector de la 
terraceria agricola, la misma que está supeditada a la disponibilidad del recurso hídrico.

El agua en la zona es captada de los deshielos de la cordillera occidental, canalizada mediante acequias hasta unos estanques ubicados en la cima de los cerros Chuatani y Muruyu. El canal principal que utilizan los pobladores de Sitajara se desprende de la cuenca del rio Yabroco o Salado, a la altura de la apacheta Yocata ubicada a 4,000 m.s.n.m. aproximadamente. En su conjunto, existen en la zona terrenos de secano activados en épocas de lluvias y terrenos de riego por gravedad.

Es probable que un gran porcentaje del área agrícola asociada al sitio Pukara sea de data prehispánica, al igual que canales y reservorios. Los sitajareños manifiestan que el abandono de los andenes ha sido paulatino y que viene desde hace muchos años atrás; algunos pobladores recuerdan y manifiestan que "desde 1940, la lluvia se malogró y que estos andenes se regaban con ella". Es obvio, el campesino se está refiriendo a un largo periodo de sequia que "malogró" el normal proceso productivo de algún sector de las terracerias; además, es obvio también entender que dicho fenómeno se haya venido reeditando desde épocas pre-hispánicas.

Los andenes que se asocian directamente al sitio arqueológico de Pukara, han sido construídos con muros de contención de $70 \mathrm{~cm}$ de altura y "banquetas" de $2.00 \mathrm{~m}$ de ancho, ligeramente inclinadas hacia el borde. No están conectados a canales de distancia, detalle que nos estaria sugeriendo su naturaleza de secano; es decir, fueron andenes regados con agua de lluvia. Sin embargo, frente a Pukara, en las laderas de la quebrada de Quilavira, las terrazas agricolas son más grandes. Ahi, los andenes están conectados a canales, tienen muros de contención de hasta 1.50 de alto y una "banqueta» de 3.00 de ancho, con planos rectos y con riego por gravedad.

En Quilavira, los andenes son utilizados permanentemente para la siembra de tuna, maiz y papa. En la parte media de la ladera, existen tres estructuras de planta circular adosadas a la pared natural de la quebrada, construidas con muros de piedra pircada unidas con barro. Las dimensiones de las tres estructuras son casi equivalentes: altura, $1.40 \mathrm{~m}$; ancho, 1.20; profundidad, $1.50 \mathrm{~m}$; espesor de los muros, $20 \mathrm{~cm}$; medidas de la "ventana" de ventilación, alto $20 \mathrm{~cm}$ por $15 \mathrm{~cm}$. de ancho. Esta "ventana" está ubicada sobre la base de la estructura,en la parte central-inferior del muro frontal.
No tiene techo, pero suponemos que originalmente lo tuvo. Por las características formales de las estructuras y su ubicación en espacios ventilados y seguros, podriamos asegurar que pertenecen a depósitos, colcas o "trojas", para almacenar productos agricolas.

\section{CAMINOS}

Por el lado SE del cerro Pukara se desplaza un camino, que es el mismo sendero que pasa por el sitio de Capanique. En el tramo asociado a Pukara, el camino tiene un ancho promedio de 3 metros; está empedrado en las cuestas mediante la construcción de gradas y muros bajos de contención en el borde externo. En las zonas planas, el camino es llano (de tierra) y mantiene cierta sinuosidad de acuerdo a la topografía de la zona. Lo importante es que este camino enlaza a varios sitios arqueológicos ubicados en los valles de Yabroco (Calofina), Susapaya (Huankarani y Kanamarca) y a los que se ubican en el trayecto hacia la zona de Candarave (Chitune, Cerro Gordo, Yucamani, Patapatani y otros).

\section{EL EQUIPO DOMÉSTICO}

Bajo este rubro se analizan las muestras recolectadas de cerámica y material lítico como batanes, ubicados en el sitio arqueológico.

La muestra de cerámica comprende 53 fragmentos de superficie, incluido el material recuperado de la limpieza de una tumba soterrada. La recolección se realizó por sectores de ocupación, considerando indicadores diagnósticos que nos permitan reconocer clases y grupos alfareros. Se recolectaron bordes, bases, asas y cuerpos con o sin decoración.

A. La primera clase: corresponde a las vasijas cerradas representadas por bordes, asas y bases de cántaros y jarras. Los cántaros se han definido a través de cinco fragmentos de bordes ( $4 \mathrm{~s} / \mathrm{d}$ y 1 $\mathrm{c} / \mathrm{d})$ de $8 \mathrm{~mm}$ de grosor y $18 \mathrm{~cm}$ de diámetro promedio; y un fragmento de base con un ligero reborde de $2.5 \mathrm{~cm}$ de grosor y $8.4 \mathrm{~cm}$ de diámetro. Las jarras, son de tamaño regular y pequeñas; los bordes y las bases recuperadas tienen decoración e indican diámetros entre los 7 a $9 \mathrm{~cm}$.

Además, se recolectáron fragmentos de bordes y asas sin decoración en los sectores habitacional y funerario. Los bordes son de labios redondeados y paredes divergentes. En el sector funerario, como resultado de la limpieza de una tumba soterrada, se recuperó dos jarras de cuerpo 
esférico, asa con inserción cuerpo-borde y base reforzada.

La alta recurrencia en la morfologia que presentan las bases, es una constante peculiar que establece la presencia de un indicador válido para la identificación de un nuevo grupo alfarero. Las bases son fuertemente reforzadas, culminando este reforzamiento en una suerte de pedestal burdo. También existen bases redondeadas, pero en casos muy aislados.

B. La segunda clase: se refiere a las vasijas abiertas, representadas por cinco tipos de cuencos:

Tipo 1, caracterizado por tener paredes convergentes, borde ligeramente plano, cuerpo esférico y un diámetro de $10 \mathrm{~cm}$ en la boca.

Tipo 2, similar al tipo 1, pero con la diferencia que éste tiene un borde inflexo redondeado; además, la convergencia de las paredes son menos pronunciadas que las del tipo anterior. Dos fragmentos de cuencos en sus extremos superiores, a la altura del borde, tienen dos pequeños apéndices con orificio a manera de falsas asas.

Tipo 3, cuencos de paredes divergentes, bordes redondeados, poco profundos y tienen un diámetro entre 12 a $16 \mathrm{~cm}$.

Tipo 4, representado por un fragmento de paredes convergentes y bordes divergentes ligeramente redondeados; la unión de ambos, dan una forma en "S" alargada. Las paredes son inflexas y de poca profundidad y el diámetro es de $12 \mathrm{~cm}$.

Tipo 5, corresponde a un cuenco con decoración interna.

El análisis macroscópico de la producción alfarera, permitió identificar cuatro grupos o alfares, tres de ellos vinculados a estilos cerámicos conocidos y el cuarto es un nuevo estilo de origen local.

\section{A. Grupo 1}

Cerámica emparentada con la alfarería del Grupo 6 de Capanique, pasta color gris y gris-rojiza, semicompacta con inclusiones de gránulos fuertes y regulares de cuarzo, arena y otros. En algunos fragmentos se observan espacios en la compactación de la pasta, ocasionados por la inclusión de material orgánico (vegetales) que a baja temperatura volatilizan. Alisamiento irregular en ambas superficies, existiendo en algunos casos las huellas dactilares del alfarero sobre la superficie externa de la vasija; a manera de engobe, se aplica una pintura roja diluída que generalmente cubre el exterior de los cuencos, mientras que el interior mantiene la coloración (café-grisáceo) propia del efecto de la cocción.

Hay algunos fragmentos dentro de este grupo, que tienen ciertas diferencias adicionales como por ejemplo: una mejor aplicación del engobe, pasta más homogénea, bordes más divergentes y bases redondeadas. Estas diferencias podrian estar respondiendo a un proceso cronológico del grupo cerámico, o en todo caso a una exigencia funcional. Sin embargo, es evidente que nos encontramos frente a una novedosa producción alfarera en Tacna, trabajada localmente por una identidad étnica que se asentó en los valles pre-cordilleranos de Tacna, durante el periodo de los Desarrollos Regionales Tardios. Otro dato importante es la alta predominancia de cerámica de este Grupo, en relación porcentual con los demás.

\section{B. Grupo 2}

Representado por cuatro fragmentos decorados, tres corresponden a jarras y el restante a un cántaro. Pasta semi-compacta de color rosáceo, con iclusiones de granos finos de cuarzo, arena y otros; alisamiento horizontal, aplicación de pintura roja-diluida sobre la superficie a manera de engobe y posterior decoración con diseños geométricos de líneas paralelas, serpenteadas y círculos concéntricos en color negro. Este grupo se vincula a la cerámica del Grupo 5 de Capanique, identificado con el estilo Pocoma de los Desarrollos Regionales Costeros Tardios.

\section{Grupo 3}

Cerámica del estilo Gentilar, que corresponde al periodo de los Desarrollos Regionales Costeros Tardios. El tratamiento alfarero es similar al aplicado en el Grupo 2, salvo algún mejoramiento en el compactado de la pasta, aplicación del decorado con la inclusión del color blanco y motivos como la voluta y el reticulado. Se recolectaron cinco fragmentos decorados: cuatro cuerpos y un borde.

\section{Grupo 4}

Representado por un solo fragmento decorado de un cuenco. Pasta color rojo-naranja, semicompacta y con temperantes de cuarzo, mica, arena y otros. Alisamiento horizontal con la posterior aplicación débil de un bruñido. Decoración interna de figuras geométricas con pintura roja- 
diluida, aplicada directamente sobre la pasta naranja de la vasija. El fragmento podría estar vinculado al denominado estilo Chilpe de los Desarrollos Regionales Tardíos, reconocido para el valle de Azapa por los investigadores ariqueños del Norte de Chile. Sin embargo, existe la posibilidad que se trate de una variante decorada de la alfarareria local de los valles pre-cordilleranos de Tacna.

Sobre el material lítico registrado, mencionaremos la existencia de batanes y morteros. Los batanes tienen una base de molienda rectangular poco profunda, con desbaste bien delineado, y mide $60 \mathrm{~cm}$ por $40 \mathrm{~cm}$. Los morteros son más pequeños, tienen la base de la molienda en forma eliptica y profunda. Se han ubicado un total de cuatro morteros en buen estado y varios fragmentados.

\section{DISCUSIÓN SOBRE EL PATRÓN DE ASENTAMIENTO}

\section{ASENTAMIENTO ARQUEOLÓGICO Y DOMINIO ESPACIAL}

Nuestras observaciones en la zona de estudio han permitido constatar la existencia de un patrón de asentamiento aldeano diferenciado en sus dos etapas de ocupación. La aldea Pukara, estaria representando contextualmente a una identidad étnica local, que nosotros la hemos denominado Estilo Sitajara, involucrada dentro del proceso de asentamientos Post-Tiwanaku para el érea Centro-Sur Andina (Lumbreras, 1974; 1981).

La aldea Pukara organiza su espacio en áreas definidas de ocupación. El hábitat es construído en puntos dominantes, sobre terrazas y zonas planas, protegido por un mecanismo de defensa tipo fosa fortificada. La idea de fortificar las aldeas, obedece a un sistema autodefensivo que es común observar en los sitios pos-tiwanaku del altiplano puneño, como una respuesta a un estado de pugnas inter-étnicas por intereses productivos. Por su parte, el área de enterramientos y la habilitación de los espacios agrícolas mediante terrazas, mantienen una unidad asociativa directa con el espacio habitacional.

Antes de la ocupación Inca y durante ésta en el valle, el patrón aldeano fortificado se diluye. Por ejemplo, la aldea de Huankarani ubicada en Susapaya, aparece delimitada por un ligero alineamiento de piedras a manera de muralla que la circundan. Huankarani está fuertemente asociada a cerámica del estilo Sitajara e Inca y se observa una estrategia de control más directo de los suelos agrícolas, por el mismo hecho que se ubica en un cerro de mediana altura y de fácil accesibilidad; todo lo contrario al emplazamiento de la aldea Pukara.

En cuanto a Capanique, éste comparte grupos cerámicos de los grupos culturales Sitajara, Pocoma e inca; evidenciando una continuidad ocupacional y/ o presencial en el sitio. Probablemente, al reocupar los Incas el sitio Capanique, reestructuraron para sus intereses el viejo modelo aldeano local, convirtíendolo al parecer en un tambo con funciones administrativas adicionales. Lamentablemente, el mal estado de las estructuras no permite dar una mayor explicación, sumándose a ello la falta de excavaciones en el sitio. Sin embargo, la alta frecuencia y densidad de cerámica Inca en la superficie, nos permite inferir la existencia de mitimaes de orígen cusqueño puestos en el valle de Sitajara para fines económicos y administrativos. Capanique, además, estaría vinculado a un camino pre-hispánico que se conecta hacia el Suroeste con las zonas de Ticaco y Tarata rumbo al valle del río Caplina; asímismo, es más que probable la vinculación que existió entre Capanique con el sitio administrativo Inca de "Sama la Antigua», ubicado al Oeste bajando por la misma cuenca.

Durante el gobierno del imperio incaico se dá el fenómeno de las concentraciones o «reducciones», en áreas accesibles, de los grupos conquistados o anexados al imperio para su control y organización, ubicándolos en puntos cercanos a redes de tráfico (Dollfus, 1981: 87-88). A pesar que para los valles occidentales la administración incaica aún no está bien definida, se presume un control y gobierno oblícuo via altiplano, construyéndose centros andminsitrativos desde donde se vuelve a capturar la red de tráfico pre-existente con las tierras altas (Núñez, 1979:20). En consecuencia, Capanique se amoldaria al modelo Inca explicado líneas anteriores; por su parte, Huankarani y Kanamarca, por su ubicación, pudieron asimilarse fácilmente a la estrategia incaica.

En definitiva, observamos un patrón generalizado de los asentamientos aldeanos en Sitajara y Susapaya, que tratan de ubicarse en las cimas de los valles de los ríos Salado (Sitajara) y Quenasani (Susapaya). El patrón se extiende en los valles cercanos como Moquegua (Stanish, 1985), Caplina y Sama (Trimborn, 1975; Gordillo, 1989; 1993), Arica (Muñoz, 1982), Camarones (Niemeyer y Schiapacasse, 1981), Tarapacá (Patricio Núñez, 1983), región del Loa Superior (Aldunate, et.al. 1986), etc. 


\section{INTERRELACIÓN ALDEANA EN EL VALLE}

Durante el periodo Sitajara (desarrollo local), representado por la aldea Pukara, observamos ciertos rasgos que nos recuerdan a tradiciones altiplánicas como mecanísmos defensivos del hábitat, tumbas soterradas encistadas y chullpas. En cuanto a la cerámica, existen sustanciales diferencias que se expresan claramente en el tratamiento de la pasta y en el acabado (monocromía: pintura roja diluida aplicada exteriormente), a diferencia del pintado negro sobre rojo e incisiones de la cerámica Kollau y del tricolor de la cerámica Allita Amaya (Tschopick, 1946; Lumbreras, 1974).

Inferimos que la aldea Pukara, durante su emergencia, en un primer momento habría recibido ciertos aportes altiplánicos, no necesariamente vía colonos. Posteriormente, hacia 1300 años d.C., Pukara se habría enmarcado dentro de un fenómeno que parece generalizarse en las zonas precordilleranas de Moquegua, al que Stanish (1985) ha denominado como la época del desarrollo de una identidad propia que establece sólo lazos económicos con el altiplano ( a través de sus investigaciones en el valle de Otora en Moquegua, Stanish define el periodo Estuquiña como un desarrollo local, que escapa a los rígidos esquemas de la verticalidad punacosta).

Colateralmente, se habría activado una constante de relaciones económicas con las poblaciones costeras Pocoma y Gentilar, mediante la inauguración de un acceso recíproco a recursos controlados en ambos espacios (costa-litoral y valles pre-cordilleranos). Pudo entonces, haberse iniciado una interrelación económica micro-regional de orden vertical dentro de la cuenca hidrográfica del valle de Sama. Por otro lado, es de suponer la existencia de un contacto con los valles pre-cordilleranos adyacentes ubicados en las cabeceras de la cuenca del río Sama (Susapaya, Yabroco, Challahuaya, Ticaco, Tarata, Tarucachi, Chucatamani, Estique, otros) y Locumba (Candarave, Cairani, Huanuara, Camilaca, otros); sectores en los cuales se han registrado aldeas similares a las de Sitajara y con el mismo tipo de cerámica local.

Hacia fines del Intermedio Tardio, el crecimiento poblacional habría obligado la creación de nuevos centros aldeanos dispersos. El incremento de los excedentes de producción es obvio. La aldea Pukara continúa siendo habitada, surgiendo otros centros como Capanique (?), Kanamarca y Huankarani (en el valle vecino de Susapaya), que se prolongan hasta la época Inca.

Ya en plena época incaica, la zona ingresa a una red de interrelaciones a gran escala, controlada a partir de centros administrativos ubicados a lo largo de su trayectoria, cuyo epicentro hegemónico más próximo era Chucuito en el altiplano puneño. La presencia Inca registrada en la cuenca del río Sama, como los sitios: Kanamarca, Huankarani, Sama la Antigua, Pampa Julia, Yalata, Morro de Sama, Punta Meca e Ite, es un claro ejemplo del control espacial que ejercieron los Incas sobre los espacios tacneños incorparados al imperio.

\section{INTEGRACIÓN POST-TIWANAKU EN EL VALLE DE SITAJARA}

Al desintegrarse la hegemonía Tiwanaku y emerger los reynos locales o regionales, se prolifera el sistema aldeano y con ello se inicia una pugna interétnica por la hegemonía de los espacios productivos y el control del recurso agua y pastizales. Se produce en el Area Centro Sur Andina, una suerte de atomización de intereses étnicos y de apropiación y control competitivo de los recursos naturales. Indudablemente, ello habría motivado la acelerada construcción de aldeas fortificadas; sobre todo, en la zona inmediata a la cuenca del Titicaca.

No sabemos casi nada sobre el impacto que generó la desarticulación Tiwanaku en los valles occidentales pre-cordilleranos; aunque, para los valles costeros surperuanos, este fenómeno puede ser explicado a través de los trabajos de Goldstein (1990). Sin embargo, el efecto debió darse bajo las mismas características sucedidas en la cuenca del Titicaca, yunga boliviana y valles costeros del surperuano y norte chileno.

En Sitajara, dado el fenómeno Tiwanaku en el área, es posible que haya existido en un primer momento un clima de desconfianza, pudiendo haber restringido en baja cuantía el tráfico tradicional entre poblaciones de costa y altura. Se habría ocasionado "... un notable empobrecimiento cultural, con escaso cuidado en la elaboración de artesania y un notable déficit de iconografia. Este proceso de simplificación cultural circum-puneño guardaría relación directa con el manejo de caravanas de corta distancia, que contactan ejes de espacios menores, alejados de los centros generadores de mayor complejidad cultural .." (ob.cit. Núñez y Dillehay, 1995: 103).

Conforme estos desarrollos locales van consolidando su estructura social y económica, a través de un fortalecimiento agrario, las actitudes de 
pugnas por los espacios productivos disminuyen y se vuelve a aperturar el tráfico interregional. La cerámica de los pueblos costeros Pocoma y Gentilar, encontrada en los sitios Pukara y Capanique, estaría validando lo inferido en las lineas precedentes. Tal vez, la aldea Pukara represente el inicio de este fenómeno post-Tiwanaku en nuestra zona de estudio.

En un segundo momento, probablemente en tiempos inmediatamente pre-Incas (1300 d.C. a 1445 d.C.?), se advierten algunas variantes en la morfología de la cerámica (cuencos de paredes divergentes y labios redondeados), con cierto parecido a los cuencos Allita Amaya (Tschopick, 1946:35); y en los modelos de asentamientos, con la presencia de estructuras circulares en las aldeas de Kanamarca y Huankarani (valle de Susapaya), vinculadas posiblemente a modelos altiplánicos Kollau. De confirmarse con mayor sustento esta presencia de rasgos, podriamos estar frente a una realidad de contactos más transparentes con grupos altiplánicos.

Pueda ser que a partir de entonces en Sitajara, se inaugure una intensificación económica y cultural a nivel micro y macro regional que derivó en un fortalecimiento de la unidad étnica y de sus relaciones de complementariedad con recursos de la costa y el litoral.

A pesar que durante el Período Tardío los Incas ocupan los sitios de Capanique, Kanamarca y Huankarani, al parecer la identidad étnica local se mantiene con ligeros cambios en la mejora del hábitat y de la producción alfarera.

\section{ASENTAMIENTOS Y MODOS DE PRODUCCIÓN}

EI indicador agrario en la zona, está sustentado por un conjunto de terrazas agrícolas asociadas directamente a los sitios arqueológicos prospectados. Es evidente el ejercicio de una economía agraria, alternada probablemente con el modelo económico de la complementariedad de recursos multi-ecológicos.

Durante el Período Sitajara (Desarrollo Local), se activó un espacio de andenes que cubria parte de los valles de Sitajara y Yabroco. Las terrazas agrícolas construídas son angostas y con muros bajos de contención, que permitieron satisfacer las exigencias del consumo local y contar con excedentes para la actividad del intercambio vía el trueque.

Durante la ocupación Inca en el valle, las tierras agrícolas se incrementaron fundamentalmente hacia la cuenca del río Quenasani y el sector de
Susapaya. Obviamente, también se extendió en las zonas de Sitajara y Yabroco. Aquí los andenes son de mayor magnitud y se decide incorporar los espacios planos al uso agrario. En consecuencia, las tierras asignadas al Estado y al Sol, habrian funcionado bajo el modelo de la mita agraria, que era una especie de prestación de servicio que se cumplia obligatoriamente en el Imperio (Rostworowski, 1988).

Otro indicador económico importante es la proximidad de los bofedales y pastizales ubicados sobre las cabeceras de la cuenca del rio Sama, en plena Puna a 4,200 m.s.n.m.. Ahí se pudo haber retomado la crianza y pastoreo de llamas y alpacas, durante los periodos Sitajara e Inca. Actualmente, Ios pobladores de Sitajara, Yabroco y Susapaya mantienen en la zona una importante actividad alpaquera.

Finalmente, la ubicación intermedia de los sitios pre-cordilleranos Pukara y Capanique con relación a los ecozonas de costa y puna, pudo haber facilitado enormemente las interrelaciones bidireccionales con poblaciones de los desarrollos costeros tardios y reynos altiplánicos. Pukara y Capanique -por ejemplo- pudieron haber asumido el papel de medios de enlace y/o filtro, entre la conección puna-costa; facilitando o dificultando el éxito de la interrelación económica entre estos dos extremos geoculturales de los Andes Centro-Sur.

\section{APROXIMACIÓN HISTÓRICA}

\section{DISCUSIÓN PRELIMINAR DE LOS RESULTADOS}

Los indicadores cerámicos permitieron plantear, para la zona de estudio, consideraciones de orden cronológico y de posibles relaciones con otros grupos de la región; por lo que pensamos que la propuesta del Estilo Sitajara es válida, en la medida que sus atributos cerámicos así lo demuestran. Rasgos como los tipos de cuencos de paredes inflexas (con o sin apéndices), forman parte de una tradición propia de los valles occidentales relacionada probablemente con los cuencos Estuquiña en Moquegua (Stanish, 1985; Lozada, 1987).

La cerámica del estilo Sitajara supervive hasta finales del período Intermedio Tardío. Durante la ocupación Inca, sufre algunos cambios leves en lo que respecta al tratamiento de la pasta y en la mayor divergencia de los bordes. Habíamos segregado (juicio preliminar) la cerámica Sitajara en dos fases: la fase I aparece con suma incidencia en la aldea Pukara, asociada superficialmente a los estilos regionales costeros Pocoma y Gentilar; mientras que la fase II se asocia frecuentemente a cerámica Inca en los sitios 


\section{Capanique, Kanamarca y HuanKarani.}

Al igual que la cerámica, en el patrón de asentamiento también vemos algunos cambios en ambos periodos. En la aldea Pukara es obvio el carácter defensivo y ubicación de altura, en donde se organiza el espacio doméstico sobre terrazas y áreas planas; en tanto, la presencia de cistas soterradas y chullpas de barro y piedra nos plantea la posibilidad de la existencia de una jerarquización o status dentro del grupo étnico

En el período Inca, los asentamientos ocupan lugares de menos altura y sin mecanismos defensivos. Continúan las estructuras sobre terrazas de fuertes muros de contención, el emplazamiento de los recintos habitacionales es más ordenado y los aparejos de los muros son más uniformes que los muros de la aldea Pukara. Además, advertimos la presencia del modelo constructivo Inca tipo "Kancha». Todas estas caracteristicas son compartidas, aunque con algunas diferencias, entre las aldeas Kanamarca y Huankarani. Sin embargo, en Kanamarca se asocian un cementerio y corrales que no aparecen en Huankarani. El cementerio tiene tres tipos de tumbas que podrian estar respondiendo a diferencias étnicas o de estatus y que su distribución en la precordillera de los valles occidentales es común; por ejemplo, las tumbas del tipo chulpiforme de Kanamarca se asemejan a las del valle de Otora-Moquegua (Stanish, 1985).

Por otro lado, la existencia de corrales en Kanamarca sustentaría la presencia de "hatos» de llamas destinadas para el tráfico caravanero y administrados por el curaca local o por un representante del Inca. Esta apreciación, podria estar relacionada con la experiencia Lupaca sobre la distribución de bienes que hace mención Gurgen Golte (1980), en donde el control de acceso a recursos de larga distancia era resuelto por los curacas. Golte, nos dice»

"... asi, los lupacas tienen acceso al ganado auquénido, a las tierras para el cultivo de papas, quinua y cañagua, a nivel de la familia nuclear (...). El acceso a los productos de otros micro-climas se da a nivel de los Kuraka..." (ob.cit. 1980:57).

Podemos inferir que la aldea Kanamarca significó el asentamiento tardio más importante en la cuenca de la confluencia de los rios Salado y Quenasani, en el contexto de los valles de Sitajara, Yabroco y Susapaya.

Uno de los aspectos que aún no se ha definido con precisión cronológica -salvo para la época Incaes el origen de las "torres funerarias" denominadas chullpas. En el altiplano la frecuencia y distribución de chullpas es impresionante; están construidas generalmente con piedras escogidas y labradas (Kidder, 1943; Tschopick, 1946; Valencia, 1983; entre otros).

La literatura sobre chullpas en los valles occidentales es escasa. En Tacna, George Squier (1974) describe algunas chullpas hechas de arcilla y paja ubicadas en la quebrada de Palca. En el año 1982, Concepción del Rio registra tres chullpas en la zona de Palca, contruidas de arcilla y paja (Informe Nro 004-82-INC-Tacna). Según Percy Dauelsberg (comunicación personal, 1988), en la sierra de AricaChile, aparecen chullpas de arcilla muy similares a las de Tacna.

José Berenguer, Carlos Aldunate y Victoria Castro, han realizado un importante trabajo sobre las chullpas de Linkán-Tocome, ubicadas en la cuenca del río Salado en la sierra ariqueña.

En nuestra zona de estudio, asociadas a la aldea Pukara, registramos tres chullpas de arcilla y paja de planta rectangular y cámara abovedada, junto a otras cinco estructuras circulares que tentativamente han sido catalogadas como chullpas. Muy cerca al poblado de Yabroco, en la prolongación Este del valle de Sitajara, existe una chullpa de planta rectangular que se levanta sobre dos plataformas de piedra; alterna el barro y la piedra en su proceso constructivo, rematando la altura en una corniza de piedras partidas. Mide $1.60 \mathrm{~m}$ de altura y está en regular estado de conservación. La cámara funeraria es de techo plano construido con troncos de queñua. La chullpa se asocia a cerámica de superficie del estilo Sitajara II e Inca. La diferencia de este tipo de chullpa con las de la aldea Pukara, es clara.

Estas chullpas de barro, paja y piedra, podrian estar marcando un sello de factura local pre-Inca e Inca en la pre-cordillera de los valles occidentales, desde el Suroeste de Arequipa hasta la sierra del norte chileno.

\section{CRITERIOS CRONOLÓGICOS}

Los viejos esquemas cronológicos para los valles occidentales, paulatinamente fueron revisados y redefinidos mediante controles estratigráficos, dataciones radio-carbónicas y por termoluminicencia THL. Se logran consolidar estilos y periodos, siendo los investigadores del norte chileno quienes mayormente aportan auspiciosos resultados. 
En la cuenca del rio Sama, el investigador alemán $\mathrm{H}$. Trimborn fecha varios sitios asociados a cerámica de los estilos costeros San Miguel, Pocoma y Gentilar cuyo rango promedio de tiempo va desde los 1,090 años d.C. hasta los 1,560 años d.C. (Trimborn, 1975; Ravines, 1982). Otros fechados tempranos, como el de 330 años d.C. en la aldea de Umapaya está por revisarse; mientras que los fechados 1,750 años d.C. y 1820 años d.C. confirman la continuidad ocupacional en los sitios Ynclán y la Vituña, respectivamente.

Los fundamentos cronológicos utilizados en nuestra zona de estudio se refieren a un trabajo comparativo de rasgos y a la asociación superficial de los indicadores observables. Por lo tanto, la propuesta cronológica que presentamos es relativa y sujeta a replanteamientos.

El estilo Sitajara sustenta una vinculación al Intermedio Tardio, sobre la base de su asociación a cerámica de los estilos Pocoma y Gentilar (1200 años d.C. a 1445 años dC. aproximadamente). Y la posterior proyección hacia el Horizonte Tardío, está indicado por su asociación superficial con la cerámica Inca. Además del indicador alfarero, podemos mencionar los patrones y modelos de hábitat y los tipos de enterramiento vinculados a tradiciones postTiwanaku e Inca.

\section{SITAJARA Y LA INTERRELACIÓN MICRO-REGIONAL}

Nos ocuparemos de los contactos de nuestra zona de estudio con las sub-regiones centro y sur de los valles occidentales. La sub-región centro incluye los valles de llo, Moquegua, Locumba, sama y Caplina desde sus cabeceras hasta el litoral. La sub-región sur, comprende desde los valles de Lluta y Azapa por el Norte, hasta Pisagua por el Sur (Ilo, 1986).

Si bien es cierto que por el momento no estamos en condiciones de definir contactos concretos entre nuestra zona de estudio y las sub-regiones mencionadas; sin embargo, podriamos adelantar algunos juicios hipotéticos. Es posible que los rasgos cerámicos Pocoma y Gentilar procedan de la cuenca media y baja del valle de Sama, activándose un mecanismo de complementariedad micro-regional de recursos por ambas partes.

El contacto con los valles de Locumba y Caplina pudieron haberse mantenido dentro del contexto macro-regional activado en los valles occidentales por las culturas de los Desarrollos Regionales Tardios, bajo las mismas propuestas de modelo económico aplicado para la cuenca del río
Sama. Este fenómeno, durante la ocupación Inca pudo acentuarse y funcionar bajo los regimenes imperiales, como el de la instauración de la Mita o prestación de servicios al Imperio.

Con la cuenca de Moquegua es probable una relación transversal con los estilos Otora y Estuquiña, ya que su cerámica y el modelo aldeano es muy similar al de Sitajara. Algo similar pudo haber sucedido con el estilo cerámico Trigalpampa, en el sector de Belen de la sierra ariqueña (Dauelsberg, 1983); asímismo, con los sectores de lquique y Camarones (Niemeyer y Schiapacasse, 1981; Muñoz, 1982; Sanhueza y Olmos, 1982; Santoro, 1983).

\section{CONTACTOS CON EL ALTIPLANO}

Para el Intermedio Tardio, aunque las relaciones poblacionales entre los valles occidentales y el altiplano son evidentes, aún no se han precisado los mecanismos interactuantes que hagan permeable su comprención. Se especula mediante el dato etnohistórico de la presunta hegemonía de control económico y político que existió por parte de los altiplánicos sobre los yungas occidentales. Maria Rostorowski nos dice al respecto:

"... la situación de los yungas del colesuyo era muy distinta al de las otras regiones costeñas del Tahuantinsuyo. La carencia de un centro de poder fuerte en los llanos hizo que, durante el Intermedio Tardio, los yungas estuvieron supeditados al dominio de los serranos del Altiplano ..." (1986:128).

Por su parte, la Visita de Chucuito hecha por Garci Diez de san Miguel en 1567, es elocuente al informar la presencia de altiplánicos Lupacas y Pacajes en Moquegua, Sama y Tarata. John Murra (1975) y Franklin Pease (1981) explican convincentemente sobre la antigua y acentuada relación entre grupos étnicos de la costa y sierra. EI mismo Rómulo Cúneo (1977:305-489). atribuye como fundadores de los valles del Caplina, Sama y Locumba a curacas de las distintas cabeceras del Reyno Lupaca.

Etnohistóricamente, Sama sustenta una presencia altiplánica hegemónica, que obedecia al modelo de complementariedad macro-regional de obtención de recursos en diversos pisos ecológicos (Murra, 1975). La definición arqueológica de este fenómeno para el Intermedio Tardio en los valles occidentales aún está en discusión.

En nuestra zona de estudio, el contacto con poblaciones altiplánicas parecen observarse en 
algunos rasgos de la arquitectura funeraria y del hábitat; sin embargo, éstos no necesariamente estarían indicando una ocupación plena en el valle. El planteamiento de Stanish $(1985 ; 1990)$ sobre una posible «independencia» de la fase Estuquiña del control altiplánico, podria estar generalizándose en los valles pre-cordilleranos del departamento de Tacna y zonas vecinas. Indudablemente, ello requiere total investigación.

En la época Inca, el control de los curacazgos de los valles occidentales fue obvio y se hizo a través de Chucuito, mediante el envío de mitimaes y administradores.

\section{LAS RELACIONES CON LA COSTA}

La explotación de las guaneras del "Morro de Sama" pudo haber motivado el inicio del contacto entre las poblaciones aldeanas agrarias precordilleranas y las costeras en la cuenca del rio Sama. No tenemos datos concretos al respecto, pero la información etnohistórica nos dice que entrada la colonia los pobladores de Tarata tenían acceso, por tradición, a la explotación de dichas guaneras (Cúneo, 1977:398). Etnográficamente, existe información sobre los itinerarios que cumplian los pobladores de los "altos de Tarata", para acceder al litoral entre Sama y Locumba (Gordillo, 1989).

Lamentablemente aún no tenemos trabajos arqueológicos para el Intermedio tardio en el contexto del "Morro de Sama", dificultando ampliar la discusión; sin embargo, algunos fragamentos recolectados por el suscrito (1987) en la Quebrada de Burros, contenían características muy similares a la cerámica del estilo Sitajara. Para el caso Inca, hemos registrado cerámica de dicha época, en los sitios de Punta Meca y Meca la Antigua, al norte del Morro de Sama y muy cerca a los bofedales o aguadas del litoral de Ite (desembocadura del río Locumba).

Es posible que los Incas ejercieran el control de la explotación de la guaneras del "Morro de Sama", del procesamiento del cochayuyo, caza de lobos marinos, pesca y extracción de peces y mariscos; y probablemente, el control del uso y explotación de las Iomas adyacentes. El registro de un camino empedrado que bordea la base del Morro de Sama -de posible data Inca- ubicado por el suscrito en 1985. indicaría la importancia de la zona como recurso económico y de estrategia vial.

EI Inca extiende su control hacia el valle medio de Sama. A la fecha hemos registrado los sitios de: Yalata, conocido también como "el aijal del Inca"
(Cavagnaro, 1988), tiene cerámica Chucuito N/R; Pampa Julia, ubicado en "Bella Vista» y Sama la Antigua (Trimborn, 1975). Todos estos sitios se encuentran dentro de la misma cuenca del Sama, a la que también pertenecen los sitios Inca registrados en nuestra zona de estudio. Es inevitable, por cierto, dejar de pensar en una tácita relación que existió entre dichos asentamientos.

\section{ALGUNAS CONSIDERACIONES FINALES DE ORDEN POLITICO Y ECONÓMICO}

Creemos que el tema que nos ocupa tiene una amplia cobertura para el análisis y discusión permanente por parte de los investigadores interesados en los períodos tardios de nuestra historia pre-hispánica. Es importante, por ejemplo, tratar la problemática de los valles pre-cordilleranos de los Andes Centro-Sur; casi nada existe al respecto.

De todas formas hay algunas propuestas sobre el tema como la hecha por Stanish (1985), que posibilita pensar en un gran horizonte cultural muy propio para los valles de pre-cordillera; sociedades que habrian activado una intensa relación económica con poblaciones de la costa y el altiplano. Esta suerte de identidad local no se ajustaria necesariamente con la tesis de Murra (1972), modelo que algunos investigadores han empleado para explicar situaciones politicas y econónicas de las culturas del Intermedio Tardio (Lumbreras, 1974 y 1981; Pease, 1981; Roswtworoski, 1986; y otros). El modelo de la verticalidad de Murra, ya lo anticipó muchos años atrás don Rómulo Cúneo Vidal (1877).

Si bien es cierto que durante la ocupación Inca los altiplánicos tuvieron acceso más continuado y fácil a los valles costeros, desconocemos hasta el momento bajo qué tipo de tratativas éstos se asentaron y convivieron con los aldeanos yungas. $\mathrm{EI}$ sitema de mitmas pudo haber sido un mecanísmo, pero no fue suficiente para generar mecanismos internos de integración y reciprocidad.

En Tacna, por ejemplo, los arqueólogos hasta la fecha no han registrado sitio alguno que evidencie una ocupación plena en los valles costeros, por parte de grupos altiplánicos del Intermedio Tardio. Solo encontramos algunos rasgos sueltos en contextos funerarios y domésticos que aparecen en asociación directa con cerámica local costera en sitios como Peañas (Gordillo y Garcia, 1989), Cristo Rey y Miraflores (Gordillo, 1989; 1993) por mencionar algunos. Cabe destacar que dichas muestras alfareras están mereciendo una revisión para descartar 
posibilidades de que se trate de cerámica precordillerana.

Para el Periodo Tardio el problema aún es más álgido. Indudablemente, debemos tratar también de entender los mecanismos de ocupación territorial bajo otras perspectivas que no sea tan sólo el análisis frio del equipo doméstico. Es importante buscar indicadores que nos lleven a entender más profundamente los modelos de ocupación territorial y

\section{REFERENCIAS BIBLIOGRÁFICAS}

ALDUNATE, CARLOS; JOSÉ BERENGUER Y OTROS. 1986 Sobre la Cronologia del Loa Superior. En: Revista Chungara Nro 16-17; Universidad de Tarapacá, AricaChile.

BIRD, JUNIUS. 1946. The cultural sequence of the Northern Chilean coast. Handbook of south American Indian 2: 558-594. Washington.

CAVAGNARO, LUIS, 1988. Materiales para la Historia de Tacna. Tomo II, Dominación Hispánica. Ed. Cooperativa San Pedro. Tacna.

CUNEO, RÓMULO. 1977. Historia de los Cacicazgos Hereditarios del Sur del Perú. En: Obras Completas, pp. 295-489. Gráfica Morsosn, Lima.

DAUELSBERG, PERCY. 1983. Investigaciones Arqueológicas en la Sierra de Arica, Sector Belén. Chungara Nro 11:6383. Universidad de Tarapacá, Arica-Chile.

DIEZ DE SAN MIGUEL, GARCI. 1964. Visita hecha a la provincia de Chucuito en 1567 Ed. cąsa de la Cultura. Lima.

DOLLFUS, OLIVER. 1981. El Reto al Espacio Andino. En: Perú Problema 20. Instituto de Estudios Peruanos. Lima.

FLORES, ISABEL. 1969. Informe preliminar sobre las investigaciones arqueológicas en Tacna. Instituto Riva-Aguero. Lima.

GOLDSTEIN, PAUL. 1985. Tiwanaku of the Moquegua Valley, Peru. A thesis submitted to, the Faculty of the Division of the Social Sciences. Departament of Antropology. Chicago, Illionis.

1990. Ocupación Tiwanaku en Moquegua. En: Gaceta Arqueológica Nro 18/19:75-104. INDEA. Lima.

GORDILLO, JESÚS. 1989. Estudio arqueológico en Sitajara, Yabroco y Susapaya: cabeceras del Valle de Sama, Dpto. de Tacna. Tesis Bachiller. Facultad de Ciencias Histórico Arqueológicas, Universidad Católica de Arequipa.

1989. Secuencia arqueológica de Tacna. En: Nueva Historia 2: 4-11. Centro de Estudios Sociales. Tacna.

1993. Catastro, inventario y evaluación de sitios arqueológicos en el valle medio del rio CaplinaTacna. Tesis Licenciatura, pp. 604. Facultad de Ciencias Histórico Arqueológicas, Universidad Católica de Arequipa.

GORDILLO, JESÚS Y MANUEL GARCIA MARQUEZ. 1989 Arquitectura funeraria $y$ análisis contextual de algunas tumbas de Peañas: breve comentario. En: de interrelación socio-económica producida entre espacios y gentes de altura y espacios y gentes de costa.

En Arica (Muñoz, 1988; Muñoz et.al. 1987; Santoro et.al.1987.) y Arequipa, se vienen contemplando la necesidad de reformular algunos criterios sobre el entendimiento del fenómeno Intermedio Tardio en los valles pre-cordilleranos. Es necesario hacerlo.

Mallku 1:2-28. INC-Tacna.

LOZADA, MARIA CECILIA. 1987. Análisis de la cerámica del componente mortuorio de Estuquiña, valle de Moquegua. Tesis Bachiller. Facultad de Ciencias Histórica Arqueológicas, Universidad Católica de Arequipa.

LUMBRERAS, LUIS GUILLERMO 1974. Los Reinos PostTiwanaku en el Area Altiplánica. Revista del Museo Nacional XL:55-85. Lima.

1981 La Arqueologia en la América Andina. Ed. Milla Batres. Lima.

1987 Exámen y clasificación de la cerámica. Gaceta Arqueológica Nro 13:3-4 y 31, INDEA. Lima.

MUELLE, JORGE C. 1969. Las cuevas y pinturas de Toquepala. Instituto Riva-Aguero, PUC. Lima.

MUÑ்Z, IVÁN. 1982. Dinámica de las estructuras habitacionales en el extremo norte de Chile (valle-costa). Chungara Nro 8:3-32. Universidad de Tarapacá, Arica, Chile.

MUÑOZ, IVÁN Y JUAN CHACAMA 1988. Cronologia por Termoluminiscencia para los Periodos Intermedio Tardio y Tardio en la Sierra de Arica. Chungara Nro 20:19-45. Universidad de Tarapacá, Arica-Chile.

MUÑOZ, IVÁN, JUAN CHACAMA Y GUSTAVO ESPINOZA. 1987. El poblamiento prehispánico Tardio en el valle de Codpa. Una aproximación a la Historia Regional. Chungara Nro 19:7-69. Universidad de Tarapacá, AricaChile.

MURRA, JOHN. 1972. El control vertical de un máximo de pisos ecológicos en la economía de las sociedades andinas. Universidad Nacional Hermilio Valdizán. Huánuco.1975 Formaciones Económicas y Políticas del Mundo Andino. Instituto de Estudios Peruanos. Lima.

NIEMEYER, Hans y Virgilio SCHAPPACASSE. 1981. Aportes al conocimiento del Periodo Tardio del extremo norte de Chile: Análisis del sector Huaracane del valle de Camarones. Chungara Nro 7. Universidad de Tarapacá, Arica Chile.

NUÑEZ, PATRICIO. 1983. Aldeas tarapaqueñas, notas y comentarios. Chungara Nio 10:29-38. Universidad de Tarapacá, Arica Chile.

NUÑEZ, Lautaro. 1979. Comentario sobre el area Centro-Sur Andina. Dpto. de Arqueología, Universidad del Norte. Antofagasta, Chile. 
NUÑEZ, LAUTARO Y TOM DILLEHAY. 1995 Movilidad giratoria, armonia social y desarrollo en los Andes Meridionales: Patrones de tráfico e interacción económica. 2da. edición. Universidad de Chile. Antofagasta

OFICINA NACIONAL DE EVALUACION DE RECURSOS NATURALES. 1976 Inventario, evaluación y uso racional de los recursos naturales de la costa: cuenca de los rios Moquegua-Locumba-Sama y Caplina. Publicación 43. Lima, Perú

PEASE, FRANKLIN. 1981 Las relaciones entre las tierras altas y la costa sur del Perú. En: Estudios Etnográficos del Perú Meridional, pp. 193-221. Ed. Masuda. Universidad de Tokio.

RAVINES, ROGGER. 1982 Panorama de la arqueologia andina Instituto de estudios Peruanos. 1ra. edición. Lima.

ROSTWOROWSKI, MARIA. 1986 La Región del Colesuyo Chungara Nro 16/17. Universidad de Tarapacá, AricaChile.

1988 Historia del Tahuantinsuyo. 1 ra edición; Instituto de Estudios Peruanos. Lima.

SANHUEZA, JULIO Y OLAF OLMOS. 1982 Usamaya I, cementerio indigena en Isluga altiplano de Iquique, I Región de Chile. Revista Chungara Nro 8. Universidad de Tarapacá, Arica-Chile.

SANTORO, CALOGERO. 1983 Camino del Inca en la Sierra de Arica. Chungara Nro 10:47-56. Universidad de Tarapacá, Arica-Chile.

SANTORO, CALOGERO; JORGE HIDALGO Y ALFONSO
OSORIO. 1987 EI Estado Inca y los grupos étnicos en el sistema de riego de Socoroma. Chungara Nro 19:71-91. Universidad de Tarapacá, Arica-Chile.

SQUIER, GEORGE. 1974 Un viaje por tierras incaicas. Capitulo XIV, por la cordillera a Tiwanaku; pp.128-146. Crónica de una expedición arqueológica (1863-1865). 1ra edición, impreso en Buenos Aires-Argentina.

STANISH, CHARLES, 1985 Economias agrarias post-Tiwanaku en la cuenca de Moquegua Manuscrito. University of Chicago, Illionis; EE.UU

1990a Economias agrarias post-Tiwanaku en la cuenca del rio Moquegua. Trabajos Arqueológico en Moquegua, Perú; Tomo 2: 115-160. Lima.

1990b Complementaridad zonal en Moquegua: Una aproximación desde el valle de Otora. Gaceta Arqueológica Nro 18/19:137-157. INDEA, Lima.

TRIMBORN, HERMANN. 1975 Investigaciones arqueológicas en los valles del Caplina y Sama (Dpto. Tacna-Perú). Studia Instituti Anthropos. Editorial verbo Divino.

TSCH@PICK, MARION H. 1946 Some notes on the Archeology of Departamente of Puno-Peru. En: Papers of Peabody Museum of American Archeology and Ethnology (27) (3). Cambrigne, EE.UU

UHLE, MAX. 1919 La Arqueologia de Arica y Tacna. Boletin de la Sociedad Ecuatoriana de Estudios Históricos 3. pp.148. Quito

VALENCIA, ALFREDO. 1983 Introducción al estudio de Sillustani. En: Arqueologia Andina pp. 45-55. Asociación de Arqueología; INC-Cusco.

CRONOLOGIA Y SECUENCIA CULTURAL TENTATIVA

cabeceras del Valle de Sama, Dpto. Tacna

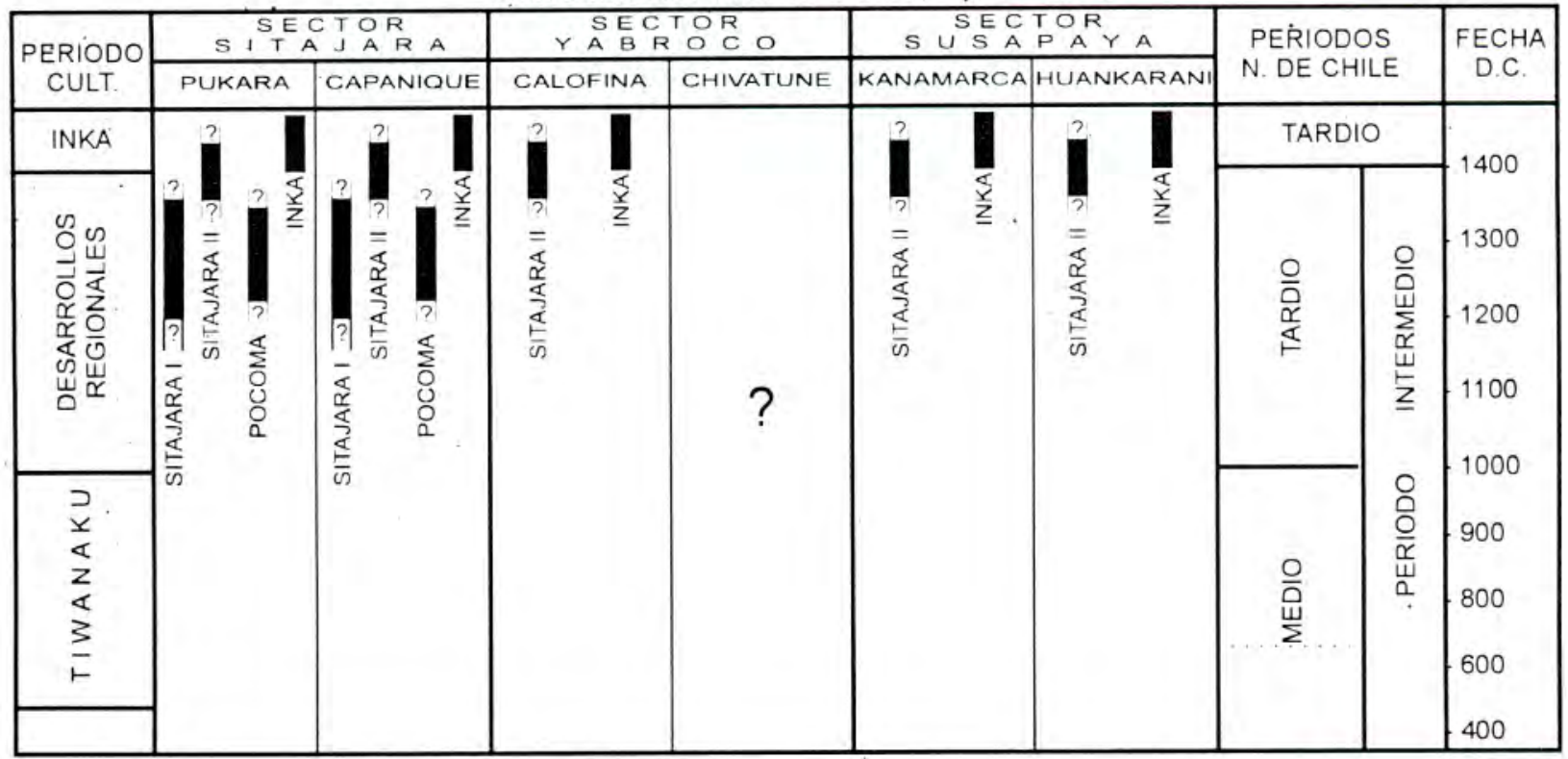

